\title{
Nonlinear meta-optics towards applications
}

\author{
Yun Zhao, Yuanmu Yang ${ }^{*}$ and Hong-Bo Sun ${ }^{*}$
}

\author{
* Correspondence: ymyang@ \\ tsinghua.edu.cn; hbsun@tsinghua. \\ edu.cn \\ State Key Laboratory of Precision \\ Measurement Technology and \\ Instruments, Department of \\ Precision Instrument, Tsinghua \\ University, Beijing 100084, China
}

\begin{abstract}
Nonlinear optical effects have enabled numerous applications such as laser frequency conversion, ultrafast electro-optical, and all-optical modulation. Both gaseous and bulk media have conventionally been used for free-space nonlinear optical applications, yet they often require complex phase-matching techniques for efficient operation and may have limited operation bandwidth due to the material absorption. In the last decade, meta-optics made of subwavelength antennas or films have emerged as novel nonlinear optical media that may potentially overcome certain limitations of bulk crystals. Due to resonant enhancements of the pump laser field as well as the use of materials with extreme nonlinearity such as epsilon-nearzero materials, meta-optics can achieve strong nonlinear responses with a subwavelength thickness. Here, we review several nonlinear optical applications, such as electric-field-induced second-harmonic generation, entangled photon pair generation, terahertz generation, all-optical modulation, and high-harmonic generation that we envision meta-optics may have distinct advantages over their bulk counterparts. We outline the challenges still faced by nonlinear meta-optics and point out some potential directions.
\end{abstract}

Keywords: Meta-optics, Nonlinear optics, Subwavelength media

\section{Springer Open}

\section{Introduction}

The field of nonlinear optics started in the 1960s, shortly after the inception of lasers [1]. It studies the nonlinear light-matter interaction under strong pump light intensities $[2,3]$. In terms of applications, to name a few, using nonlinear optical effects such as second-harmonic generation (SHG), third-harmonic generation (THG), differencefrequency generation (DFG), and high-harmonic generation (HHG), people have extended the operation band of coherent light sources spanning from the extreme ultraviolet (EUV) to the terahertz $(\mathrm{THz})$ regime. In addition, nonlinear processes such as spontaneous parametric down-conversion (SPDC) and four-wave mixing (FWM) can be used to generate single photon or entangled photon pair for quantum optics applications.

For the variety of nonlinear optical applications, it is first critical to identify the proper material with a large effective nonlinear susceptibility, ultrafast response, as well as tunability, for efficient and on-demand harmonic generation, as well as all-optical modulation.

(c) The Author(s). 2021 Open Access This article is licensed under a Creative Commons Attribution 4.0 International License, which permits use, sharing, adaptation, distribution and reproduction in any medium or format, as long as you give appropriate credit to the original author(s) and the source, provide a link to the Creative Commons licence, and indicate if changes were made. The images or other third party material in this article are included in the article's Creative Commons licence, unless indicated otherwise in a credit line to the material. If material is not included in the article's Creative Commons licence and your intended use is not permitted by statutory regulation or exceeds the permitted use, you will need to obtain permission directly from the copyright holder. To view a copy of this licence, visit http://creativecommons.org/licenses/by/4.0/. 
Furthermore, the nonlinear medium often needs to satisfy the phase-matching condition. Phase mismatch has been a long-standing issue faced by the nonlinear optics community. It is typically described by the momentum mismatch $\Delta k$, which originates from the dispersion of the nonlinear materials and the consequent refractive index mismatch of the fundamental and harmonic light that propagates inside the nonlinear medium. If $\Delta k \neq 0$, the newly generated nonlinear signals become out-of-phase with earlier ones, and the phase difference depends on the nonlinear interaction length $L$. The nonlinear conversion efficiency reaches a local maximum when $L$ is equal to the odd multiple of the coherence length $L_{c}=\frac{\pi}{\Delta k}$. Without proper phase-matching, the generated harmonic light may destructively interfere with each other, thus significantly lowering the nonlinear conversion efficiency. To date, the most commonly adopted phase-matching methods include birefringent phase-matching (BPM) and quasi-phase-matching (QPM) [4-7]. However, the BPM method can be limited by the finite birefringence of the nonlinear medium. It is only applicable for a certain incident angle. The QPM method requires a relatively complex fabrication process for nonlinear domain inversion. Both phase-matching techniques typically work only for a specific pump wavelength. This greatly limits the bandwidth of certain nonlinear optical devices. For example, for the $\mathrm{THz}$ wave generated by intra-pulse DFG or optical rectification, the bandwidth of the generated $\mathrm{THz}$ wave is largely limited by the phase-matching conditions [8]. For the generation of entangled photon pair in birefringent or periodically poled crystals, whether using SPDC or FWM, it is difficult to achieve the efficient emission of entangled photon pair over a large spectral bandwidth $[9,10]$.

The absorption of nonlinear optical materials at the fundamental and harmonic wavelengths is another common problem. For HHG in solids, as the photon energy in the ultraviolet band can be higher than the bandgap of most nonlinear optical materials, most of the generated signal is absorbed [11-13]. For THz waves generated by nonlinear processes, their bandwidth is also limited by the absorption caused by various vibrational and rotational modes of the nonlinear medium [14].

In recent years, there has been a growing interest to study nonlinear light-matter interaction in subwavelength-thick meta-optics [15-27]. The meta-structures typically support resonances that can significantly boost the pump or the harmonic light field. Furthermore, due to the subwavelength light-matter interaction length $L$ in the metastructures with $L<L_{c}$, the phase-matching requirements can be greatly alleviated, and the material absorption may be largely mitigated, as schematically shown in Fig. 1a. These combined effects may thus significantly boost the meta-structures' nonlinear optical response in comparison with the same amount of materials without resonant enhancements.

Metasurfaces are composed of arrays of optical antennas with a subwavelength thickness [28-30]. The optical antennas can be metallic ones that support localized surface plasmon resonances or dielectric ones that support Mie resonances, thus significantly boosting the local field [31-35]. Apart from the field enhancements, metasurfaces can also allow flexible control of the amplitude, phase, and polarization of the light, leading to nonlinear wavefront control $[36,37]$.

Recently, there has also been a surge in the interest in the extreme nonlinear optical properties of subwavelength-thick thin films such as transparent conductive oxides (TCOs) or layered thin films that have an epsilon-near-zero (ENZ) response [38-52]. 


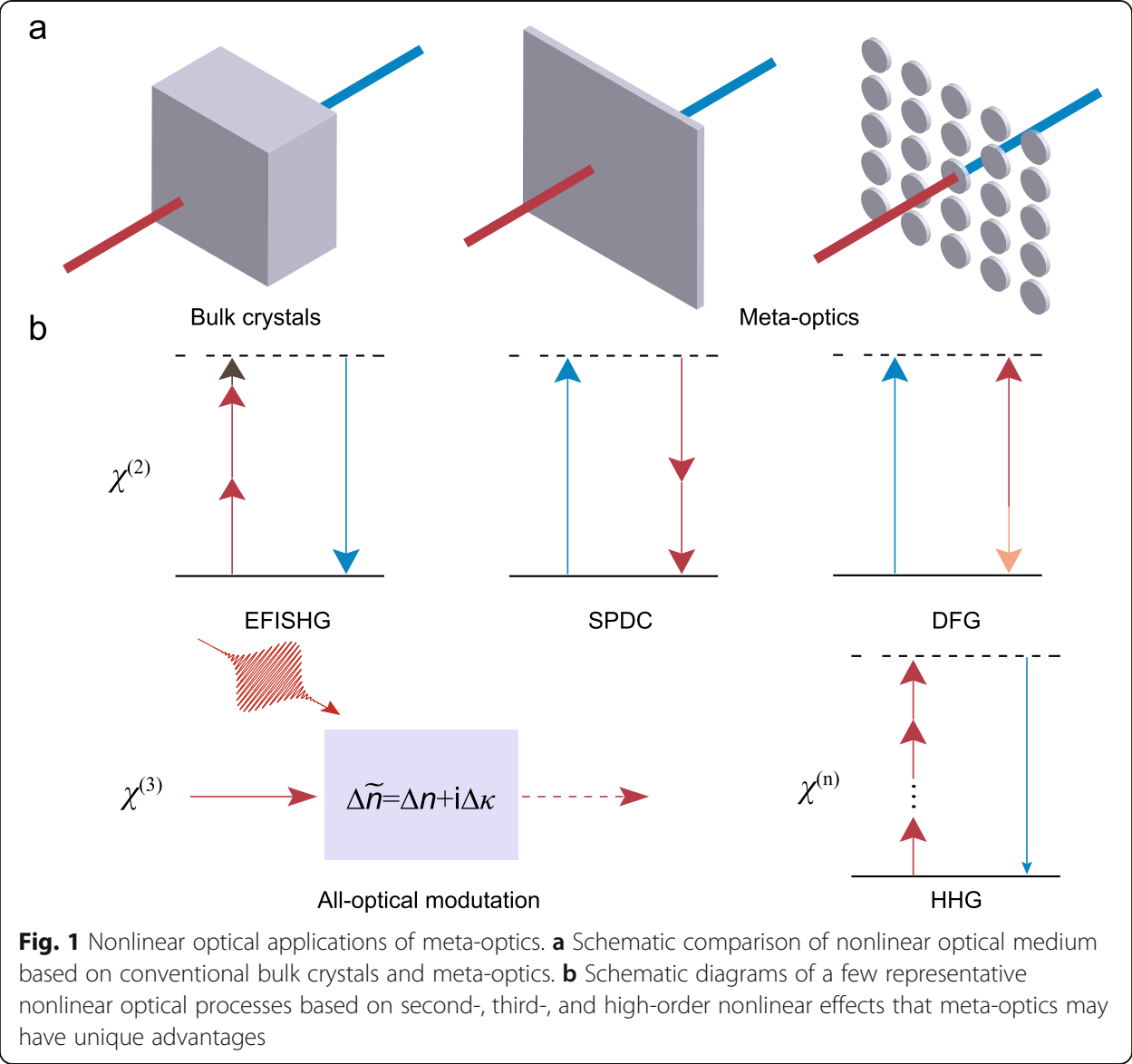

In the ENZ region where the real part of the permittivity of a material vanishes, according to the electromagnetic boundary condition, a strong field enhancement can be obtained inside the ENZ thin film with a subwavelength thickness, under the condition that the material loss is moderate [53-55]. Around the ENZ wavelength, the nonlinear refractive index $n_{2}=\frac{3 X^{(3)}}{4 n_{0} \operatorname{Re}\left(n_{0}\right) \varepsilon_{0} c}$ can be extremely large as a result of the small $\operatorname{Re}\left(n_{0}\right)$ $[56-58]$. Here, $X^{(3)}$ is the third-order nonlinear susceptibility, $n_{0}$ is the linear refractive index, $\varepsilon_{0}$ is the vacuum permittivity, and $c$ is the speed of light. Besides the large effective nonlinear susceptibility, ENZ thin films made of TCOs may have a subpicosecond response time for all-optical modulation, much faster than that of noble metals and semiconductors $[59,60]$.

To date, most work on nonlinear meta-optics focuses on frequency conversionrelated applications, such as SHG and THG. We refer the readers to several excellent review papers on these topics [61-69]. Despite the large enhancement of optical nonlinearity, due to the short light-matter interaction length of meta-optics, their SHG and THG conversion efficiencies still need to be drastically improved to be comparable or even exceed that of the bulk crystals.

In this review, we focus on a variety of less-discussed nonlinear optical applications that are beyond the scope of SHG and THG, in which we find meta-optics may have unique advantages. We categorize these nonlinear optical applications based on their nonlinear order, as illustrated in Fig. 1b. For second-order nonlinear optical 
applications, we focus on electric-field-induced second-harmonic generation (EFISHG), entangled photon pair generation, and $\mathrm{THz}$ generation. For the third-order nonlinear meta-optics, we focus on ultrafast all-optical modulation, with specific attention on their applications in laser Q-switching and mode-locking. We then further discuss the use of meta-optics for applications in coherent extreme ultraviolet pulse generation by HHG. In each of these applications, we will review the respective merits and current limitations of meta-optics. We will also discuss potential methods for further improving the nonlinear conversion efficiency of meta-optics, and outline a few possible development directions.

\section{Second-order optical nonlinearity}

Second-order optical nonlinearity has been of vital importance for extending the operation band of coherent light sources with frequency conversion processes such as SHG, DFG, and SPDC. Meta-optics may provide a unique opportunity for the active control of second harmonic signals via EFISHG. As the reverse process of SHG, SPDC based on meta-optics may be useful for the multi-channel entangled photon pair generation. Meta-optics can be also used as broadband nonlinear $\mathrm{THz}$ sources through the intrapulse DFG process.

\section{Electric-field-induced second-harmonic generation}

SHG is prohibited in bulk crystals with inversion symmetry. Such a requirement can be alleviated in the EFISHG process, as it is an effective second-order optical process with third-order nonlinear susceptibility [70]. The nonlinear polarization can be described by $P(2 \omega) \propto \chi^{(3)} E_{\mathrm{dc}} E_{\omega} E_{\omega}$, where $P(2 \omega)$ is the nonlinear polarization at frequency $2 \omega, E_{\mathrm{dc}}$ and $E_{\omega}$ are the static electric field and the optical field at the pump frequency $\omega$. The effective second-order susceptibility $X_{\text {eff }}^{(2)}$ can be described by $X_{\text {eff }}^{(2)}=X^{(3)} E_{\mathrm{dc}}$. For conventional bulk crystals, a high voltage is required to obtain a large $E_{\mathrm{dc}}$, and consequently a large $X_{\text {eff }}^{(2)}$. On the other hand, meta-structures can greatly reduce the voltage requirement due to their subwavelength thickness. For example, an electric-fieldinduced nonlinear modulation at the applied voltage of $10 \mathrm{~V}$ is obtained in a plasmonic metasurface, as shown in Fig. 2a [71]. The metasurface consisting of a periodic Au hole array and a silver film, separated by a 100 -nm-thick $\mathrm{Al}_{2} \mathrm{O}_{3}$ layer, is designed to have a large resonantly enhanced electric field at around $810 \mathrm{~nm}$. By tuning the applied voltage from 0 to $10 \mathrm{~V}$, the normalized second harmonic signal shows a linear dependence with the applied voltage, with a modulation depth up to $9 \%$. Here, the modulation depth is defined as $\Delta I_{2 \omega} / I_{2 \omega}$ where $\Delta I_{2 \omega}$ is the change of second-harmonic intensity with an applied electric field, and $I_{2 \omega}$ is the minimum second-harmonic intensity. A large modulation depth of the second-harmonic signal may benefit applications such as signal processing. Later, Lee et al. demonstrate EFISHG in a silicon-based metasurface [72]. The schematics of the metasurface unit cell as well as the experimental result, are shown in Fig. 2b. The metasurface consists of silicon grating with 190-nm-thickness and standard $\mathrm{Cr} / \mathrm{Au}$ electrode. The modulation depth reaches up to $12 \%$ at the metasurface's magnetic Mie resonance wavelength of $785 \mathrm{~nm}$, with an applied voltage of 10 $\mathrm{V}$. This work expands the application of silicon-based second-order nonlinear optical devices and reveals a way for the active modulation of the nonlinear signal with a 
a

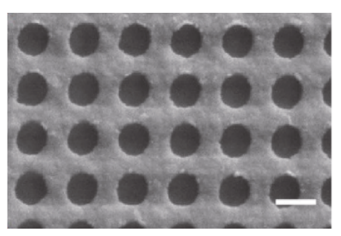

b

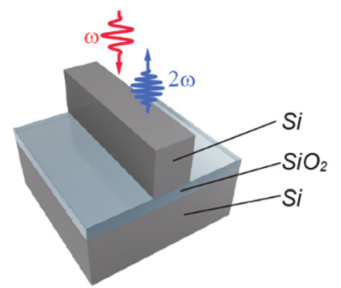

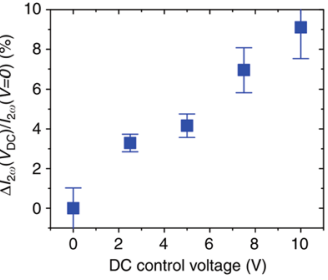

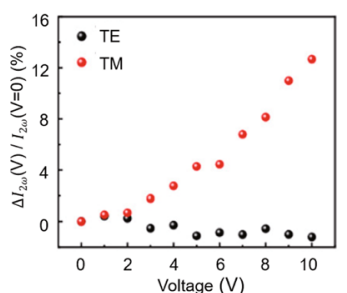

C
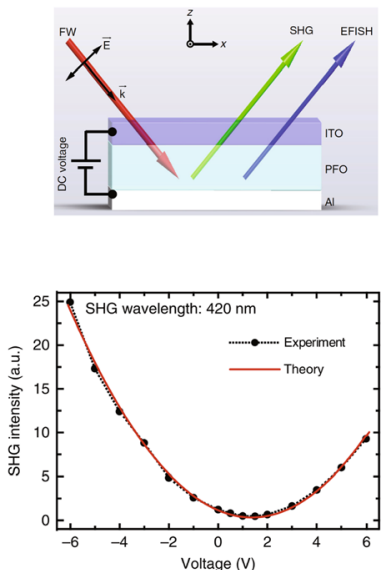

Fig. 2 Meta-optics for EFISHG. a Left: scanning electron microscopy (SEM) image of the plasmonic metasurface consists of Au hole array. Right: the dependence of the modulation depth on the applied DC voltage [71]. b Left: schematic image of the dielectric metasurface consists of silicon grating that supports Mie resonance. Right: the dependence of the modulation depth on the applied voltage under the excitation with the TM- (red) and TE- (black) polarized light [72]. c Top: schematic of EFISHG in an ITO/PFO/ Al metafilm. Bottom: the dependence of the SHG intensity on the applied voltage [73]

subwavelength spatial resolution. Even more efficient modulation may be obtained in unstructured metafilms. Chen et al. demonstrate electrically controlled SHG from a 100-nm-thick polymer thin film sandwiched between a bottom ITO layer and a top aluminum layer, as shown in Fig. 2c [73]. The polymer is a blue-emitting material with a bandgap energy of $2.95 \mathrm{eV}$ and has a large value of $\chi^{(3)}$ at near-infrared wavelengths [74]. It is found that there is a resonance enhancement of nonlinearity when the energy of second-harmonic light is close to the absorption edge of the polymer. With the enhancement from the band-edge effect, a giant modulation depth of $~ 1899 \%$ is obtained at a pump wavelength of $840 \mathrm{~nm}$ (the SHG photon energy is $3.18 \mathrm{eV}$, close to the material bandgap), with an applied voltage of only $6 \mathrm{~V}$. This extremely large modulation depth results from the minimum value of SHG intensity at an applied voltage of $1.5 \mathrm{~V}$ due to the contribution of the surface $\chi^{(2)}$.

\section{Entangled photon pair generation}

The generation of entangled photon pair is a fundamental building block for the quantum communication network [75-78]. SPDC is the most commonly used method for the generation of entangled photon pair. However, the current SPDC sources are used under phase-matching conditions such as $\beta$-barium borate $[79,80]$, and periodically poled $\mathrm{KTiOPO}_{4}[81,82]$, which limits the spectral correlation width to a few nanometers [82]. The efficient generation of entangled photon pair in a broad spectral width may be desired in future high-capacity quantum communication networks.

Due to the subwavelength thickness of meta-structures, the allowed $\Delta k$ may be extremely large, which results in the emission of photon pair with a broad spectrum. The first demonstration of SPDC in a thin film with $\mu \mathrm{m}$ scale thickness is reported by Okoth et al. [83]. As shown in Fig. 3a, SPDC with about $500 \mathrm{~nm}$ spectral width is obtained in a $6-\mu \mathrm{m}$-thick $\mathrm{LiNbO}_{3}$ layer. The authors further predict that micro- or nano- 

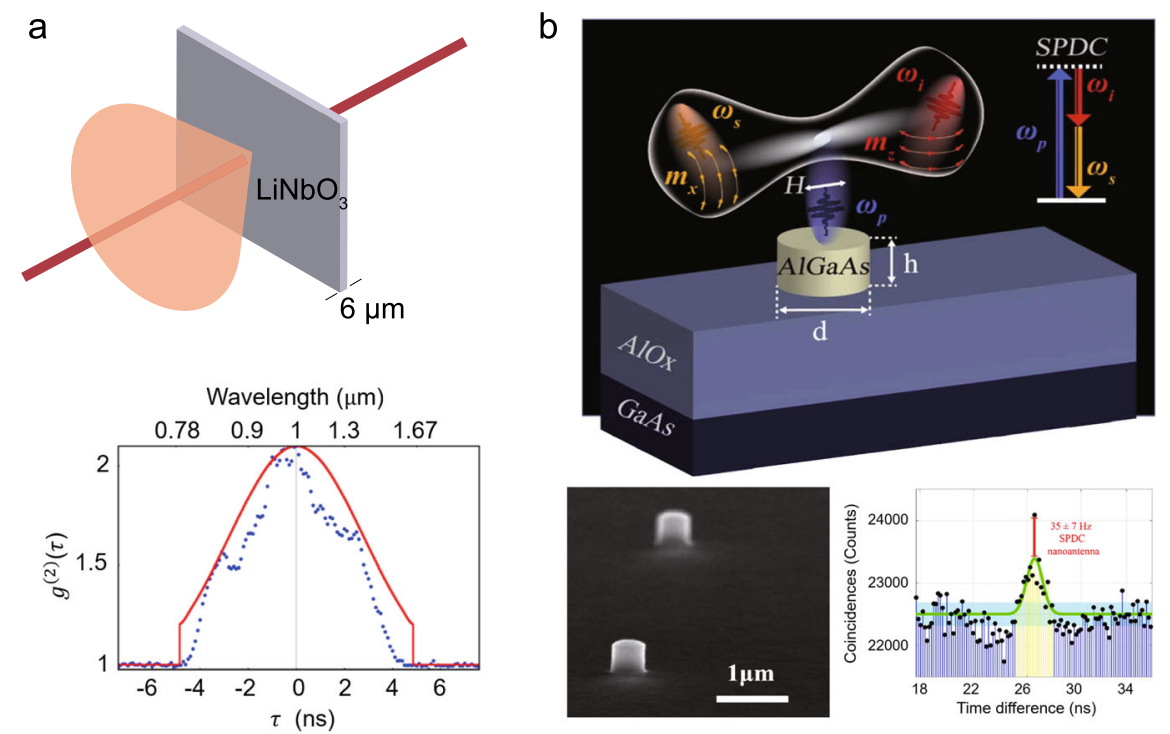

Fig. 3 Meta-optics for SPDC. a Top: schematic of SPDC in a $\mathrm{LiNbO}_{3}$ thin film. Bottom: the frequency spectrum measured using the single-photon spectroscopy method. The red curve is the calculated frequency spectrum and the blue dots are the values of second-order correlation as a function of the delay time [83]. b Top: schematic of SPDC in a single AIGaAs disk nanoantenna which supports Mie resonances. Bottom: SEM image of the antenna (left) and the measured coincidences and the fitted Gaussian curve (right) [84]

scale SPDC sources may promote high-order nonlinear processes such as the generation of the three-photon state which are challenging in bulk nonlinear crystals due to the large refractive index difference between the pump and the signal light. In 2019, Marino et al. report an SPDC source from an AlGaAs disk nanoantenna which supports Mie resonances at both the pump and the signal/idler wavelengths, as shown in Fig. 3b [84]. Due to the relatively slow generation rate of SPDC, the polarization correspondence is first investigated through the sum-frequency generation (SFG) process according to the SFG-SPDC correspondence [85, 86]. The SPDC measurement is then demonstrated after optimizing the experimental parameters with the polarization of horizontal-polarized pump beam and verticallypolarized bi-photon beams. A photon-pair generation rate of up to $35 \mathrm{~Hz}$ is obtained with a pump laser wavelength of $785 \mathrm{~nm}$ and with an average pump power of $2 \mathrm{~mW}$. This rate is about one order of magnitude higher than conventional photon pair sources based on bulk crystals, when normalized to the pump energy in the nanoantenna. The bandwidth of the generated photon pair spectrum is expected to be about $150 \mathrm{~nm}$ owing to the use of the relatively broad magneticdipole resonance. The phase-mismatch-free photon pair sources based on metaoptics with a broad spectral correlation width may provide a new avenue for highcapacity multi-channel quantum entanglement and may be a suitable platform to observe higher-order parametric down-conversion effects. Currently, the efficiency of SPDC using meta-optics still needs to be further improved, for example, by using materials with higher nonlinear susceptibility and resonant structures with stronger field enhancements. 


\section{Broadband THz wave generation}

$\mathrm{THz}$ waves, with a frequency ranging from $0.1-10 \mathrm{THz}$, have been found attractive for a variety of applications including wireless communication [87, 88], imaging [89-91], security [92], and sensing [93, 94]. However, the lack of $\mathrm{THz}$ sources has largely hindered its development. Particularly, for $\mathrm{THz}$ spectroscopy applications, an ultrabroadband $\mathrm{THz}$ source can be highly desired. Among other methods such as photoconductive switching, broadband $\mathrm{THz}$ source has been routinely generated from intrapulse DFG or optical rectification by impinging a femtosecond laser onto a nonlinear crystal with second-order nonlinearity. For a transform-limited Gaussian pump pulse, the bandwidth of the emitted $\mathrm{THz}$ signal $\Delta v$ is partly determined by the pulse duration of the pump laser $\tau_{\mathrm{p}}$ as $\Delta v=0.44 / \tau_{\mathrm{p}}$. Furthermore, the phase mismatch of the nonlinear crystal poses a major limitation of the generated $\mathrm{THz}$ bandwidth [95, 96]. Most materials with large second-order nonlinearity may have a drastically different refractive index at the optical and $\mathrm{THz}$ spectral domain. For example, $\mathrm{LiNbO}_{3}$ has a refractive index of 2.25 at the wavelength of $800 \mathrm{~nm}$, and a refractive index of 4.90 at the frequency of $1 \mathrm{THz}$. Therefore, to achieve phase-matching, complicated techniques such as tilted pulse front $[97,98]$ need to be applied. Other nonlinear materials with smaller second-order nonlinear susceptibility of $\chi^{(2)}$, such as ZnTe and GaP, may have a smaller refractive index difference at the optical and $\mathrm{THz}$ regime and can be phase-matched at a given combination of the pump (infrared) and the parametric (THz) wavelength. However, the material dispersion still restricts their generated $\mathrm{THz}$ bandwidth typically below $3 \mathrm{THz}$ when the crystal thickness is above $1 \mathrm{~mm}$.

Nonlinear meta-optics may be a potential solution to the $\mathrm{THz}$ bandwidth problem since meta-structures can be free from the phase-matching requirement. In 2014, Luo et al. reported a metasurface-based broadband THz source [99]. The metasurface, composed of an array of split-ring resonator (SRR) on the ITO glass substrate, is shown in Fig. 4a. The SRR supports a magnetic-dipole resonance at the wavelength of $1500 \mathrm{~nm}$. By pumping it with a femtosecond laser with a central wavelength at $1500 \mathrm{~nm}$, a broadband $\mathrm{THz}$ signal, with frequency ranging from $0.1-4 \mathrm{THz}$, is generated. The origin of $\mathrm{THz}$ emission from the metasurface is attributed to intraband DFG induced by the broken symmetry of the second-order nonlinear current distribution. The broad $\mathrm{THz}$ bandwidth is only limited by the pulse duration of the pump laser (140 fs) and may be further extended. More impressively, the $\mathrm{THz}$ conversion efficiency of the metasurface is found to be comparable with a bulk, 1-mm-thick ZnTe crystal with identical pump laser parameters, despite the thickness of the metasurface is only on the order of tens of nanometers. Later, a more efficient broadband $\mathrm{THz}$ emission from a dark-modeassisted metasurface is theoretically proposed, with 2 order of magnitude efficiency enhancement in comparison with conventional SRR-based metasurfaces [101]. The metasurface consists of two gold SRRs with identical orientation, which are located asymmetrically on two sides of a thin silicon plate and blocked by a gold wall periodically. The designed structure has a largely reduced radiative damping, thus has a much larger local field enhancement, resulting in a further improved $\mathrm{THz}$ generation efficiency. In 2019, spatiotemporally tailored terahertz emission is experimentally demonstrated in a metasurface. By periodically changing the SRR's orientation, as shown in Fig. 4b, a single-cycle Hermite-Gauss wavepacket is generated [100]. More recently, a broadband $\mathrm{THz}$ source as a result of surface optical rectification in a $19 \mathrm{~nm}$-thick ITO 
a

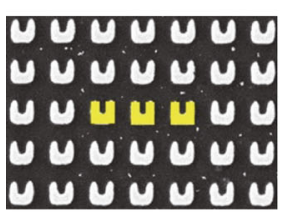

b

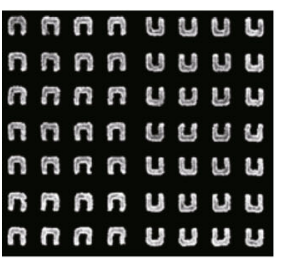
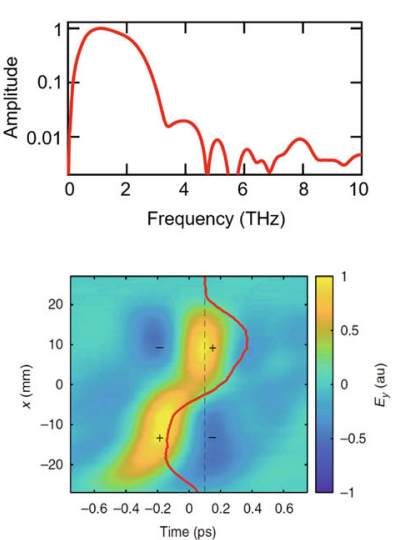

C
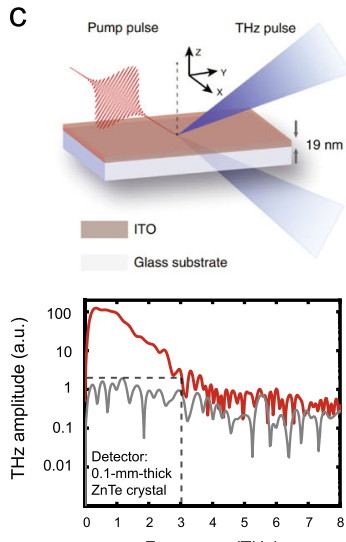

Frequency $(\mathrm{THz})$

Fig. 4 Meta-optics for broad THz wave generation. a Left: SEM image of the metasurface consists of SRR array. Right: spectrum of the THz wave [99]. b Left: SEM image of the metasurface consists of two oppositely oriented SRR array. Right: spatiotemporal profile of the single cycle Hermite Gauss wavepacket [100]. c Top: schematic of THz wave generation from an ITO metafilm. Bottom: THz spectrum generated from ITO (red) and the noise spectrum (grey) [45]

ultrathin film has been demonstrated, as shown in Fig. 4c [45]. With the large field enhancement caused by the ENZ effect, THz generation is greatly boosted when operating near its ENZ wavelength. The bandwidth of the $\mathrm{THz}$ generation can be over 3 $\mathrm{THz}$, only limited by the pulse duration of the pump laser and the phase-matching requirement of the ZnTe detection crystal. In comparison with bulk nonlinear crystals, nonlinear meta-structures may generate $\mathrm{THz}$ waves with broader bandwidth and designer wavefront, with a conversion efficiency not too far behind.

\section{Third-order optical nonlinearity}

THG and FWM, the main third-order optical nonlinear processes, are commonly used for applications such as frequency conversion in laser systems, and frequency comb generation [102, 103]. The change of nonlinear refraction index caused by self- or cross-phase modulation is the basis of all-optical modulation. Meta-structures with resonances, owning to their ultrafast modulation speed and large modulation depth, can be particularly suitable for all-optical modulation. Here we focus the discussion on their use for applications in laser Q-switching and mode-locking.

\section{All-optical modulation}

All-optical modulation has recently regained a lot of interest for applications in highspeed data processing and neuromorphic computing [104, 105]. Under the stimulus of the pump light, the refractive index $n$ depend on the pump intensity $I$ as $n=n_{0}+n_{2} I$. The induced refractive-index change $\Delta \tilde{n}=n_{2} I$ can modulate the phase, intensity, and polarization of the signal light. For most nonlinear materials, the $\Delta \tilde{n}$ is small, leading to a limited device modulation depth. Meta-optics with strong resonances can result in a large electric field enhancement and a consequent large effective $\Delta \tilde{n}$. Therefore, a large modulation depth under low energy consumption may be obtained. Furthermore, the demand for ultrafast modulation requires a short recovery time of $\Delta \tilde{n}$. 
For most semiconductor-based all-optical modulation devices, $\Delta \tilde{n}$ results from the electron excitation from the valence band to the conduction band. The slow recombination rate of electron-hole pairs often limits the modulation time to tens of picoseconds [105-107]. As a recent example, a large reflection modulation at picosecond timescale was demonstrated in a GaAs-based dielectric metasurface, as shown in Fig. 5a [108]. The structure consisting of GaAs nanopillars is designed to support Mie resonance. Under a low pump fluence of $310 \mathrm{uJ} / \mathrm{cm}^{2}$, a relative modulation depth from $-50 \%$ to $73 \%$ is observed and an absolute modulation of up to 0.35 is obtained at the magneticdipole resonance. For metal and TCOs, the all-optical modulation can be based on both interband and intraband excitation of free carriers. The response time of the intraband excitation depends on the energy exchange rate between electrons and phonons [114]. The modulation time of metal is typically on the order of several picoseconds [115, 116]. For example, a transmission modulation with picosecond response time is demonstrated in the plasmonic metasurface consisting of gold nanorods, as shown in Fig. $5 \mathrm{~b}$ [109]. Using the nonlocality to enhance the nonlinearity, $80 \%$ transmission change is obtained under a pump fluence of $7 \mathrm{~mJ} / \mathrm{cm}^{2}$. Later, Ren et al. report a $40 \%$ relative transmission modulation in a gold metasurface made of an asymmetric SRR array [117]. With the local field enhancement supported by the plasmonic closed mode, the required pump fluences of $270 \mu \mathrm{J} / \mathrm{cm}^{2}$ are reduced by about one order of magnitude in comparison with that in gold nanorods. Utilizing the much faster two-photon absorption process, the modulation time can be shorter than $100 \mathrm{fs}$.

Contrary to plasmonic metasurfaces, TCOs have a smaller electron heat capacity which allows a fast energy exchange rate between electrons and phonons, leading to a modulation time of hundreds of femtoseconds. In 2016, an absolute transmission control at a subpicosecond timescale in the ITO metasurface is reported by Guo et al. (Fig. 5c) [110]. The ITO nanorods array, which supports localized surface plasmon resonances, enables an absolute modulation depth over $20 \%$ in both near-infrared and midinfrared spectral range. In addition to metasurfaces, unstructured TCOs films can also be used for ultrafast all-optical modulation with a large modulation depth. For example, an ultrafast reflectance modulation of up to $40 \%$ is reported in AZO metafilms [118]. Due to the defect-enhanced recombination process caused by the low-temperature fabrication procedure, the modulation time is below 1 ps. Later, Clerici et al. further extended the functionality of AZO by combining the intraband excitation and interband excitation via a two-color laser field [111]. As shown in Fig. 5d, the subpicosecond modulation is observed. This work shows that AZO metafilm can dynamically control the modulation bandwidth and the transmitted probe wavelength by the combination of the two excitation effects. The all-optical modulation depth can be further improved by using ENZ materials with higher electron mobility and by using more judiciously engineered optical resonances. As shown in Fig. 5e, Yang et al. report femtosecond optical polarization switching in indium-doped CdO-based heterostructures [112]. Due to the large electron mobility and low optical loss, a high Q-factor Berreman-type absorber is designed at the ENZ wavelength. Under a resonant excitation, an absolute reflectance modulation over $80 \%$ and a polarization modulation with an extinction ratio of 91 are obtained within 800 fs. The large nonlinear responses of TCOs typically only occur in a relatively narrow bandwidth. The combination of ENZ materials and metasurfaces can overcome this constraint. In 2018, a coupled structure combining ITO film 
a

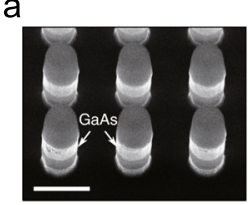

C

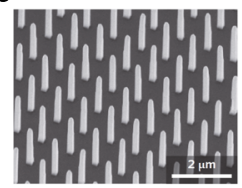

e
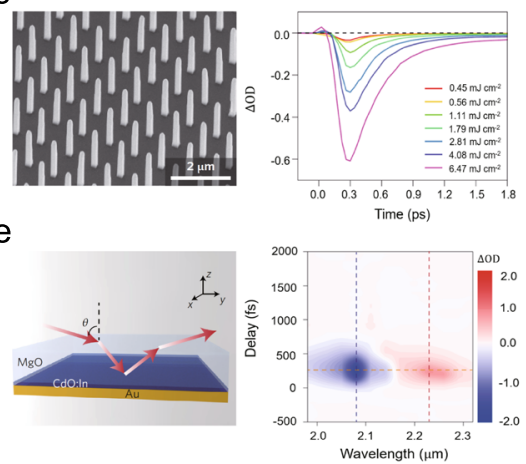

b

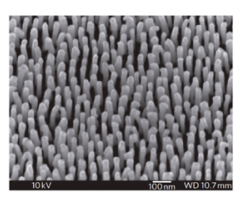

d

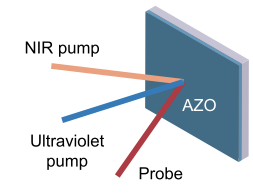

$f$

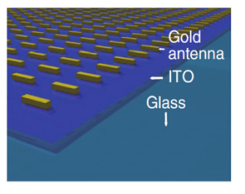

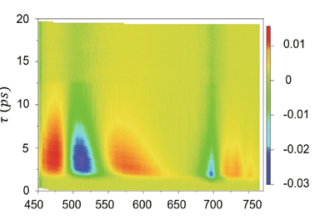
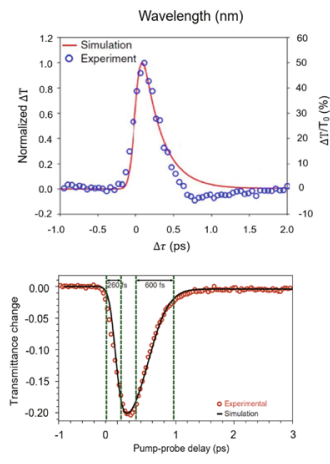

Fig. 5 Meta-optics for all-optical modulation. a Left: SEM image of the GaAs metasurface. Right: measured differential reflectance map as a function of the wavelength and delay time. The red and blue colour scales correspond to positive and negative changes, respectively [108]. b Left: SEM image of the plasmonic metasurface. Right: optical density $(\triangle \mathrm{OD})$ map as a function of the wavelength and delay time. The red and blue colour scales correspond to positive and negative changes, respectively [109]. c Left: SEM image of the ITO metasurface. Right: the dependences of $\triangle \mathrm{OD}$ on the delay time with different pump fluences [110]. $\mathbf{d}$ Left: schematic of transmission modulation from an aluminum-doped zinc oxide metafilm. Right: changes in transmission as a function of the delay time [111]. e Left: schematic of polarization switching from a CdO based metafilm absorber. Right: $\triangle O D$ map as a function of the wavelength and delay time. The red and blue colour scales correspond to positive and negative changes, respectively [112]. f Left: schematic of the structure composed of a plasmonic metasurface coupled to an ITO film with an ENZ response. Right: changes in transmission as a function of the pump-probe delay time [113]

to plasmonic metasurfaces is reported by Alam et al. [113]. As shown in Fig. 5f, the strong coupling between the localized surface plasmon resonance and the ENZ mode provides a large nonlinear refractive index change of \pm 2.5 over a broad spectral range of $200 \mathrm{~nm}$ with subpicosecond recovery time. Therefore, meta-optics, especially those based on TCOs materials, not only provide a large modulation depth but also exhibit faster modulation speed, with potential applications for low-power, ultrafast all-optical modulation in high-density nonlinear nanophotonic devices.

\section{Laser Q-switching and mode-locking}

All-optical modulation and saturable absorption are third-order nonlinear effects widely used for laser Q-switching and passive mode-locking. The two typical processes involved in all-optical modulation and saturable absorption are shown in Fig. 6a. Saturable absorption describes the effect that the absorption coefficient $\alpha$ of the material given by $\alpha=\frac{\alpha_{0}}{1+I / I_{s}}$. Here, $\alpha_{0}$ is the linear absorption coefficient, and $I_{s}$ is the saturation intensity. Saturable absorption typically results from the imaginary part of $\chi^{(3)}$ and can exist in most light-absorbing materials. The saturable absorption performance of a material is usually determined by the nonlinear absorption coefficient $\beta=\frac{\alpha-\alpha_{0}}{I}=\frac{6 \omega}{\varepsilon_{0} c^{2} n_{0}^{2}} \mathrm{Im}$ $\left(\chi^{(3)}\right)$. Conventional saturable absorbers are made of semiconductor materials [121$125]$, although they may suffer from the relatively long response time of tens of picoseconds limited by the interband electron-hole recombination process. In recent years, 

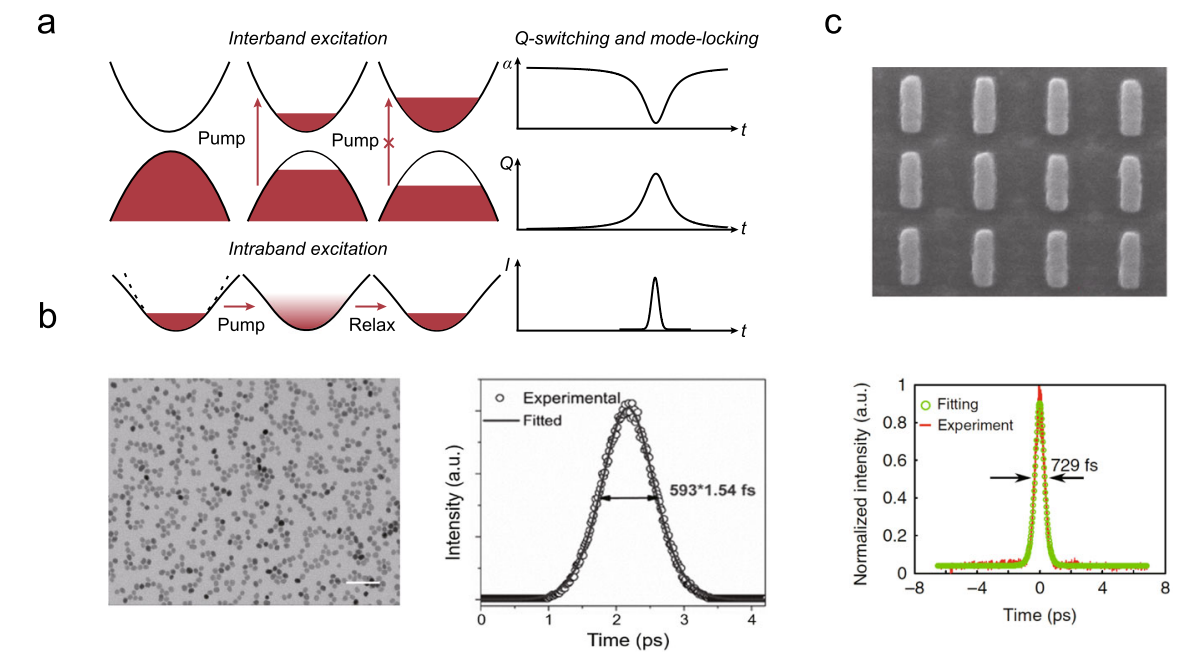

Fig. 6 Meta-optics for laser Q-switching and mode-locking. a Illustration of the processes of saturable absorption based on either interband or intraband excitation (left) and the principle of laser Q-switching and mode-locking (right). b Left: transmission electron micrograph of the ITO nanocrystal. Right: temporal width of one pulse [119]. c Top: SEM image of the plasmonic metasurface. Bottom: temporal width of single soliton [120]

there has been a growing interest to use two-dimensional materials such as graphene [126-129], $\mathrm{MoS}_{2}$ [130-132], and black phosphorus [133-135] as saturable absorbers, partly due to their ultrafast modulation time of up to a few hundred femtoseconds. However, the relatively low damage threshold and low modulation depth may be a limiting factor for their practical applications. Meta-optics based on metals and TCOs may provide an alternative approach for laser Q-switching and mode-locking, due to the ultrafast modulation time on the picosecond or sub-picosecond time scale, as well as the large modulation depth enhanced by resonances. Using ITO nanocrystals as the saturable absorber, a laser pulse with $593 \mathrm{fs}$ temporal width can be obtained (Fig. 6b) [119]. The ITO nanocrystals exhibit response time within $450 \mathrm{fs}$ and modulation depth up to $160 \%$ in the ENZ region. More recently, a demonstration of laser mode-locking in saturable plasmonic metasurfaces is reported [120]. As shown in Fig. 6c, a 729 fs laser pulse is generated in a fiber laser cavity with plasmonic metasurface as the saturable absorber when pumping at a resonant wavelength of $1550 \mathrm{~nm}$. The modulation depth of $60 \%$ is far greater than that of other saturable absorbers, yet the exact mechanism of such a large modulation depth has not been reported. Furthermore, compared with the conventional semiconductor saturable absorber, plasmonic and ENZ materials can operate in a wide frequency region, with the relatively easy tuning of their resonance wavelengths by varying the cavity design or the carrier density.

\section{Higher-order optical nonlinearity}

HHG is a high-order nonlinear optical process that is often nonperturbative and occurs when the laser field strength is comparable with the atomic bonding strength $[3,136-$ 138]. HHG forms the basis of the generation of coherent soft X-ray and EUV pulses and has been widely used for time-resolved photon-electron spectroscopy and isolated attosecond pulse generation [139-142]. HHG is conventionally observed in rare gas atoms [143-145], with relatively low efficiency. In 2008, leveraging the local field enhancement induced by the plasmonic resonance of a bowtie antenna array, Kim et al. 
reported the enhanced HHG in argon gas in the vicinity of the bowtie antenna [146]. In 2011, HHG is first observed in a solid-state $\mathrm{ZnO}$ crystal [147]. Bulk solids typically have a higher efficiency than gases because of their higher atom density [148, 149]. However, owing to the strong absorption for the generated signals whose photon energy is higher than the bandgap of nonlinear materials, the efficiency of HHG in solid is still limited, and a high laser pump fluence is required.

Meta-optics with subwavelength propagation length can reduce the pump threshold with the resonantly-enhanced pump laser field. In 2016, Han et al. utilize the extreme light confinement of a metal cone structure to enhance HHG from sapphire, as shown in Fig. 7a [150]. With the field enhancement supported by surface plasmon polaritons, high harmonics up to the 13th order are generated when pumped with a $75 \mathrm{MHz}$ laser oscillator of $0.1 \mathrm{TW} / \mathrm{cm}^{2}$ intensities. Later, HHG from a plasmonic metasurface is reported [151]. The structure composed of an array of Au antenna on a 500-nm-thick crystalline silicon substrate is shown in Fig. 7b. When the polarization of the pump laser is parallel to the long axis of the antenna, with the consequent excitation of the electric-dipole resonance of the antenna, the intensities of generated high harmonics are enhanced by 5 to 10 times. However, the small overlap between the electric field and nonlinear media may limit the overall enhancement of HHG. Moreover, metallic antennas typically have a low laser damage threshold. Low-loss dielectric metasurfaces supporting Mie resonance with a larger field confinement volume may benefit HHG with higher generation efficiency and laser damage. In 2018, enhanced nonperturbative HHG from silicon metasurfaces is reported by Liu et al., as shown in Fig. 7c [152]. With the high-Q Fano resonance, the harmonic emission can be enhanced by two orders of magnitude in comparison with a bare silicon film of the same thickness. The odd harmonics from the 5th to 11th order are observed when the laser polarization is set to excite the Fano resonance. At an excitation intensity of $0.071 \mathrm{TW} / \mathrm{cm}^{2}$, the 5 th harmonic is enhanced over 30 times and the conversion efficiency is about $5 \times 10^{-9}$. More recently, Yang et al. report the HHG from indiumdoped CdO when pumped at its ENZ wavelength, as shown in Fig. 7d [153]. Under the ppolarized illumination at an incident angle of $50^{\circ}$, the high-harmonic up to the 9th order (corresponding to the wavelength of $250 \mathrm{~nm}$ ) is observed. The required peak pump intensity of $14.1 \mathrm{GW} / \mathrm{cm}^{2}$ is much weaker than a peak pump intensity on the order of $1 \mathrm{TW} /$ $\mathrm{cm}^{2}$ that is typically required for observing HHG in bulk crystals. While the diffraction of the radiated high harmonics may make the collection of high harmonic signals from metasurfaces difficult and inefficient, a carefully engineered metasurface can be used to tailor the wavefront of generated high-harmonic signals. In 2018, Sivis et al. demonstrated controlled high-harmonic emission patterns from $\mathrm{ZnO}$ or silicon metasurfaces with a Fresnel zone plate pattern, as shown in Fig. 7e [154]. Compared with bulk crystals, metastructures can be good candidates as HHG sources with low pump threshold and controllable wavefront. Currently, the efficiency and cut-off frequency of HHG from solids are limited by a low damage threshold. The use of wide bandgap materials to construct metasurfaces may further improve their damage threshold.

\section{Conclusions}

Here, we have reviewed the use of meta-optics for several nonlinear optical applications. To summarize, we have found that subwavelength nonlinear media may be especially useful when the following merits are highly desired: 


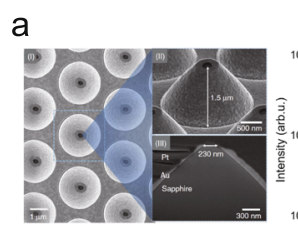

C

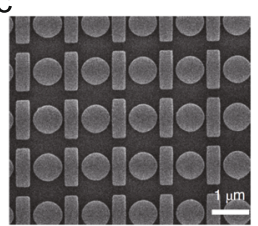

e
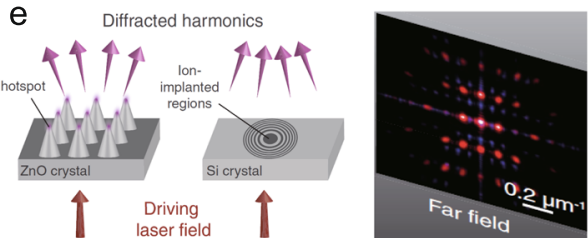

b
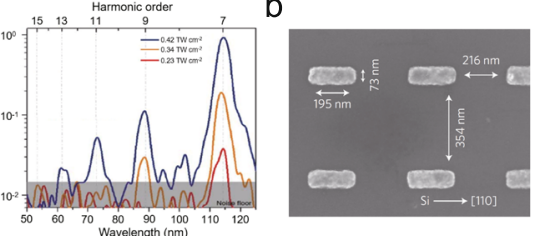

d
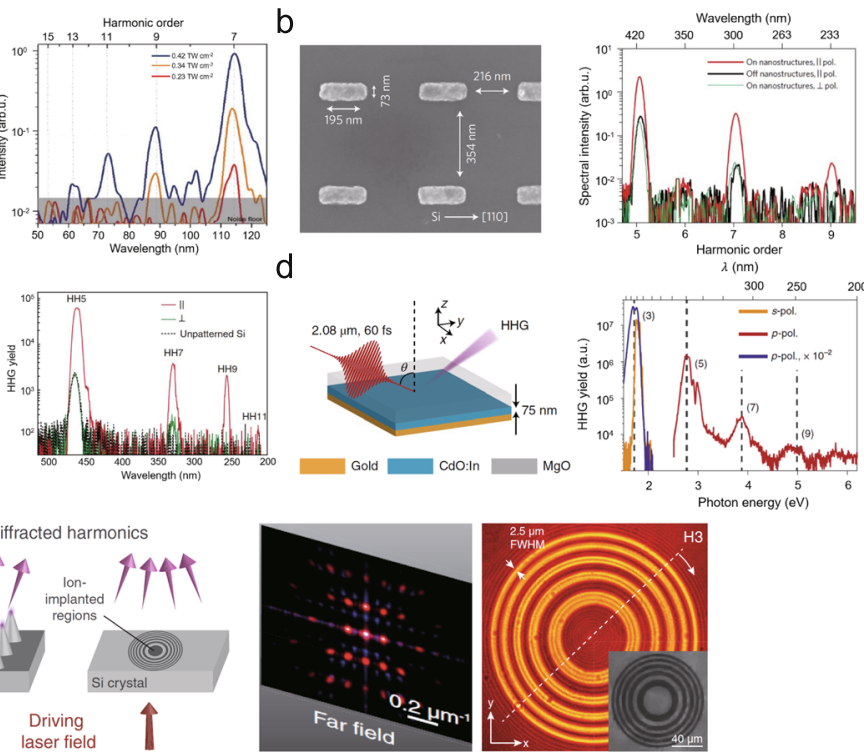

Fig. 7 Meta-optics for HHG. a Left: SEM image of the metal-sapphire nanostructures. Right: the measured EUV spectra with different pump-laser intensity [150]. b Left: SEM image of the plasmon-assisted metasurface composed of Au nanoantenna array on a silicon substrate. Right: the high-harmonic spectrum of the antenna array with the polarization parallel (red) and perpendicular (green) to the long axis of the antennas, and high-harmonic spectrum of the unpatterned Si substrate (black) [151]. c Left: SEM image of the silicon metasurface on a sapphire substrate that supports high-Q Fano resonance. Right: the highharmonic spectra when the polarization of excitation pulses is parallel (red) and perpendicular (green) to the bar. The dotted black line is the emission from unpatterned Si [152]. $\mathbf{d}$ Left: schematic of HHG from a CdO-based metafilm with an ENZ response. Right: the spectrum extends from 3rd to 9th under p-polarized (red and blue) and s-polarized (orange) excitation [153]. e Left: illustration of nanostructured and ionimplanted semiconductors that generate tailored high-harmonic wavefronts. Middle: the far-field diffraction of the third (red) and fifth (blue) harmonics. Right: the third-harmonic emission pattern [154]

1). Broad spectral bandwidth. While phase-matching is a pre-requisite for bulk nonlinear crystals, typical phase-matching techniques such as BPM and QPM can only work at one specific wavelength at a time. On the contrary, for meta-optics, their subwavelength thickness causes them to be free from the phase-matching requirement, allowing one to potentially sacrifice some efficiency to gain the bandwidth (Fig. 8a). This feature may be particularly useful for broadband $\mathrm{THz}$ generation and entangled photon pair generation.

2). Ultrafast response. The electron-hole recombination time of semiconductors is generally greater than tens of picoseconds. On the other hand, intraband transition in metals and TCOs typically take place on a time scale of several picoseconds and a few hundred femtoseconds, respectively, therefore they can be particularly suitable for ultrafast all-optical switching at a THz rate (Fig. 8b). Combining the ultrafast material dynamics with tailored resonances for an enhanced modulation depth, nonlinear meta-optics may find applications in laser Q-switching and modelocking. Additionally, the ultrafast saturable absorber may be useful for nonlinear activation in the all-optical neuromorphic circuit.

3). Wavefront control. While the wavefront of generated nonlinear signals from bulk crystals is typically uniform, metasurfaces with tailored spatial antenna arrangement can tune the polarization, phase, and amplitude of light at the subwavelength spatial resolution, thus achieving wavefront control of nonlinear 


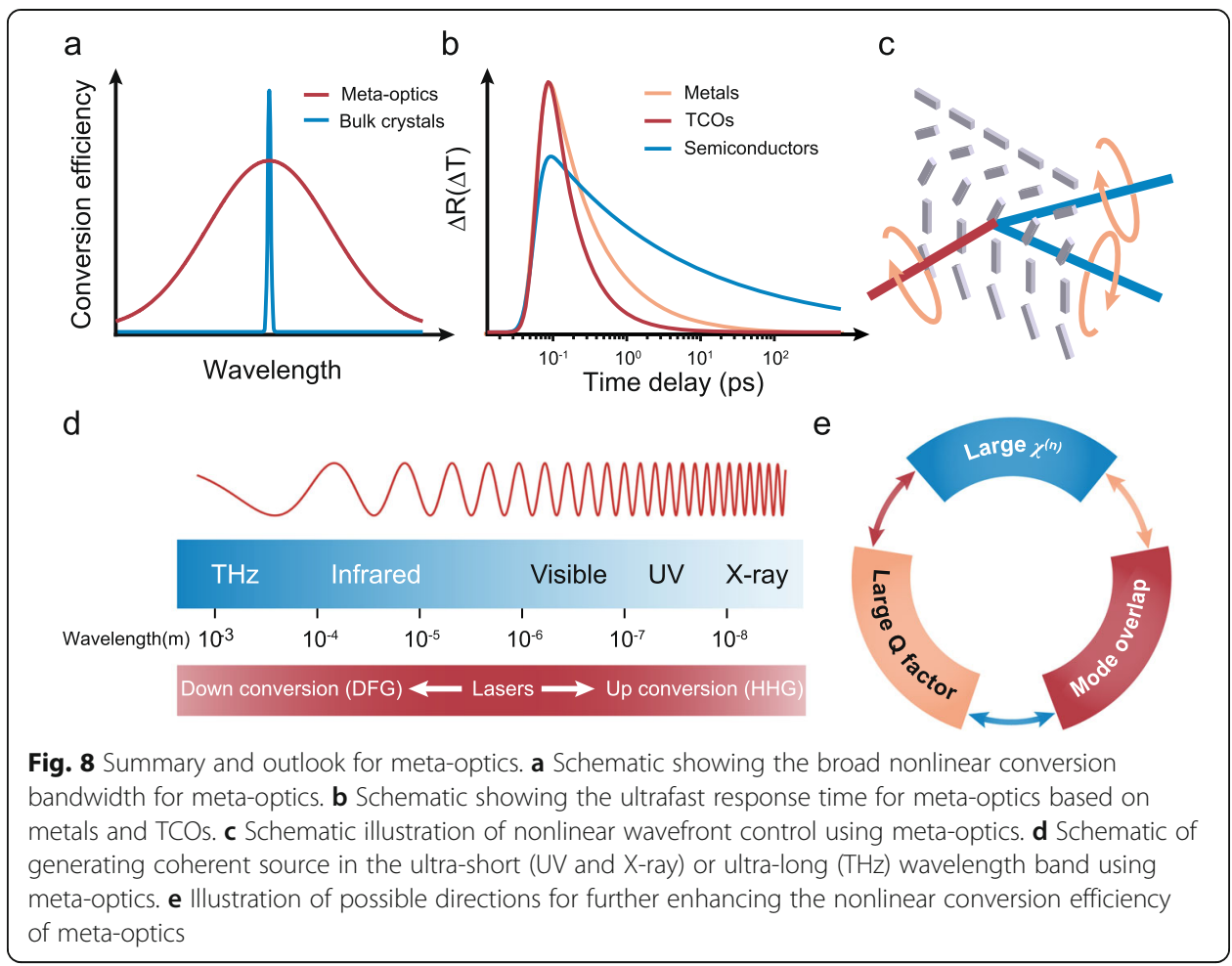

signals (Fig. 8c). Electro-optical modulation, for example, based on metal-insulatormetal metasurfaces may have potential applications in beam steering and holograph.

4). Operation in the typically absorbing band. The absorption of nonlinear optical materials induced by electronic or phononic transitions is a major problem that limits the operation band for bulk nonlinear crystals. Meta-structures, with their subwavelength thickness, may greatly alleviate the issue, allowing efficient HHG and $\mathrm{THz}$ generation in the typically absorbing ultra-short (EUV or soft X-ray) or ultra-long (mid-infrared and THz) wavelength bands (Fig. 8d).

Notably, despite some resonant enhancements, the nonlinear conversion efficiency of many meta-optical devices is still rather low, mostly due to the subwavelength nonlinear light-matter interaction length, which is still the major limitation towards practical applications. Here, we outline several routes to enhance the nonlinear conversion efficiency, as schematically shown in Fig. 8e. Firstly, one can explore options to integrate materials with a large intrinsic nonlinear susceptibility for use in meta-optics. For instance, $\mathrm{LiNbO}_{3}$ is one of the most widely used nonlinear materials with a large intrinsic nonlinear susceptibility, a low optical loss over a wide bandwidth, as well as a relatively high laser damage threshold. In recent years, we have seen the rapid progress of lithium niobate on insulator technology [155-161], which may enable the development of nonlinear meta-optics based on $\mathrm{LiNbO}_{3}$ with largely improved efficiency. Another strategy to enhance the nonlinear conversion efficiency is to design the resonator to support extremely high- $\mathrm{Q}$ resonances, for example, by leveraging the concept of Fano resonances and bound states in the continuum [162-164]. Finally, the meta-structures may be designed to be resonant at both the pump and signal wavelength, with good modal 
overlap. This can be achieved by designing the meta-structures via inverse design techniques such as the adjoint method and deep learning [165-168]. Finally, the advancement of novel fabrication techniques may inspire a more complex design of metaoptics. For instance, using resonant plasmonic antennas to enhance the local field, the femtosecond laser can lead to subwavelength ablation and reshaping, resulting in a selforganized meta-optic structure with high spatial resolution $[169,170]$. The recent development of femtosecond laser-based three-dimensional (3D) fabrication technology has allowed sub-20-nm spatial resolution [171], which provides additional degrees of freedom to create 3D meta-structures based on a variety of material platforms towards nonlinear optics applications $[172,173]$. This technology has recently been used to fabricate nonlinear photonic crystals allowing phase-matching over all three dimensions [174].

Nonlinear meta-optics is still at an early stage of development. With its unique merits not readily available in conventional bulk crystals, as well as the still largely unexplored physics, materials, and optical cavity designs, we envision many more exciting results towards practical applications in the imminent future.

\footnotetext{
Abbreviations

SHG: Second-harmonic generation; THG: Third-harmonic generation; DFG: Difference-frequency generation; HHG: Highharmonic generation; EUV: Extreme ultraviolet; THz: Terahertz; SPDC: Spontaneous parametric down-conversion; FWM: Four-wave mixing; BPM: Birefringent phase-matching; QPM: Quasi-phase-matching; TCOs: Transparent conductive oxides; ENZ: Epsilon-near-zero; EFISHG: Electric-field-induced second-harmonic generation; SEM: Scanning electron microscopy; SFG: Sum-frequency generation; SRR: Split-ring resonators; 3D: Three-dimensional
}

\section{Acknowledgements}

Not applicable.

\section{Authors' contributions}

Yuanmu Yang and Hong-Bo Sun provided an outline and guided writing. Yun Zhao wrote the manuscript and prepared the figures. The author(s) read and approved the final manuscript.

\section{Funding}

This work is supported by the National Natural Science Foundation of China (NSFC) (61975251).

Availability of data and materials

Data sharing is not applicable to this article as no new data were created or analysed in this study.

\section{Declaration}

\section{Competing interests}

The authors declare that they have no competing interests.

Received: 21 January 2021 Accepted: 5 March 2021

Published online: 16 April 2021

\section{References}

1. Franken PA, Hill AE, Peters CW, Weinreich G. Generation of optical harmonics. Phys Rev Lett. 1961;7(4):118-9.

2. Shen YR. The principles of nonlinear optics. New York: Wiley; 1984

3. Boyd RW. Nonlinear optics. 3rd ed. New York: Academic; 2008

4. Fejer MM, Magel GA, Jundt DH, Byer RL. Quasi-phase-matched 2nd harmonic-generation-tuning and tolerances. IEEE J Quantum Electron. 1992;28(11):2631-54.

5. Yamada M, Nada N, Saitoh M, Watanabe K. First-order quasi-phase matched $\mathrm{LiNbO}_{3}$ waveguide periodically poled by applying an external field for efficient blue second-harmonic generation. Appl Phys Lett. 1993;62(5):435-6.

6. Myers LE, Eckardt RC, Fejer MM, Byer RL, Bosenberg WR, Pierce JW. Quasi-phase-matched optical parametric oscillators in bulk periodically poled LiNbO . J Opt Soc Am B-Opt Phys. 1995;12(11):2102-16.

7. Armstrong JA, Bloembergen N, Ducuing J, Pershan PS. Interactions between light waves in a nonlinear dielectric. Phys Rev. 1962;127(6):1918-39. https://doi.org/10.1103/PhysRev.127.1918.

8. Blanchard F, Sharma G, Razzari L, Ropagnol X, Bandulet H-C, Vidal F, Morandotti R, Kieffer JC, Ozaki T, Tiedje H, Haugen $H$, Reid M, Hegmann F. Generation of intense terahertz radiation via optical methods. IEEE J Selected Topics Quantum Electron. 2011;17(1):5-16. https://doi.org/10.1109/JSTQE.2010.2047715.

9. Yan H, Chen J. Narrowband polarization entangled paired photons with controllable temporal length. Sci China-Phys Mech Astronomy. 2015:58(7):1-10

10. Couteau C. Spontaneous parametric down-conversion. Contemp Phys. 2018;59(3):291-304. 
11. He R, Lin ZS, Zheng T, Huang H, Chen $C T$. Energy band gap engineering in borate ultraviolet nonlinear optical crystals: ab initio studies. J Phys-Condens Matter. 2012;24(14):145503.

12. Wu M, Ghimire S, Reis DA, Schafer K, Gaarde MB. High-harmonic generation from Bloch electrons in solids. Phys Rev A. 2015;91(4):043839.

13. Ghimire S, Reis DA. High-harmonic generation from solids. Nat Phys. 2019;15(1):10-6.

14. Tani M, Fukasawa R, Abe H, Matsuura S, Sakai K, Nakashima S. Terahertz radiation from coherent phonons excited in semiconductors. J Appl Phys. 1998;83(5):2473-7.

15. Klein MW, Enkrich C, Wegener M, Linden S. Second-harmonic generation from magnetic metamaterials. Science. 2006; 313(5786):502.

16. Aouani H, Rahmani M, Navarro-Cia M, Maier SA. Third-harmonic-upconversion enhancement from a single semiconductor nanoparticle coupled to a plasmonic antenna. Nat Nanotechnol. 2014;9(4):290-4.

17. Celebrano M, Wu X, Baselli M, Grossmann S, Biagioni P, Locatelli A, et al. Mode matching in multiresonant plasmonic nanoantennas for enhanced second harmonic generation. Nat Nanotechnol. 2015;10(5):412-7. https://doi.org/10.1038/ nnano.2015.69.

18. O'Brien K, Suchowski H, Rho J, Salandrino A, Kante B, Yin XB, et al. Predicting nonlinear properties of metamaterials from the linear response. Nat Mater. 2015;14(4):379-83.

19. Segal $\mathrm{N}$, Keren-Zur $\mathrm{S}$, Hendler $\mathrm{N}$, Ellenbogen $\mathrm{T}$. Controlling light with metamaterial-based nonlinear photonic crystals. Nat Photonics. 2015;9(3):180-4.

20. Palomba S, Zhang S, Park Y, Bartal G, Yin XB, Zhang X. Optical negative refraction by four-wave mixing in thin metallic nanostructures. Nat Mater. 2012;11(1):34-8.

21. Ren MX, Plum E, Xu J, Zheludev NI. Giant nonlinear optical activity in a plasmonic metamaterial. Nat Commun. 2012;3:833.

22. Lee J, Tymchenko M, Argyropoulos C, Chen PY, Lu F, Demmerle F, et al. Giant nonlinear response from plasmonic metasurfaces coupled to intersubband transitions. Nature. 2014;511(7507):65-9.

23. Chong KE, Staude I, James A, Dominguez J, Liu S, Campione S, et al. Polarization-independent silicon metadevices for efficient optical wavefront control. Nano Lett. 2015;15(8):5369-74.

24. Li GX, Chen SM, Pholchai N, Reineke B, Wong PWH, Pun EYB, et al. Continuous control of the nonlinearity phase for harmonic generations. Nat Mater. 2015;14(6):607-12.

25. Almeida E, Bitton O, Prior Y. Nonlinear metamaterials for holography. Nat Commun. 2016;7:12533.

26. Li G, Zentgraf T, Zhang S. Rotational doppler effect in nonlinear optics. Nat Phys. 2016;12(8):736-40

27. Ellenbogen T, Voloch-Bloch N, Ganany-Padowicz A, Arie A. Nonlinear generation and manipulation of airy beams. Nat Photonics. 2009;3(7):395-8.

28. Kildishev AV, Boltasseva A, Shalaev VM. Planar photonics with metasurfaces. Science. 2013;339(6125):1232009.

29. Meinzer N, Barnes WL, Hooper IR. Plasmonic meta-atoms and metasurfaces. Nat Photonics. 2014:8(12):889-98

30. Yu N, Capasso F. Flat optics with designer metasurfaces. Nat Mater. 2014;13(2):139-50.

31. Linden S, Niesler FBP, Forstner J, Grynko Y, Meier T, Wegener M. Collective effects in second-harmonic generation from split-ring-resonator arrays. Phys Rev Lett. 2012;109(1):6488-92.

32. Shcherbakov MR, Neshev DN, Hopkins B, Shorokhov AS, Staude I, Melik-Gaykazyan EV, Decker M, Ezhov AA, Miroshnichenko AE, Brener I, Fedyanin AA, Kivshar YS. Enhanced third-harmonic generation in silicon nanoparticles driven by magnetic response. Nano Lett. 2014;14(11):6488-92. https://doi.org/10.1021/nl503029j.

33. Kruk S, Weismann M, Bykov AY, Mamonov EA, Kolmychek IA, Murzina T, et al. Enhanced magnetic second-harmonic generation from resonant metasurfaces. Acs Photonics. 2015;2(8):1007-12.

34. Yang Y, Wang W, Boulesbaa A, Kravchenko BDP II, Puretzky A, et al. Nonlinear Fano-resonant dielectric metasurfaces. Nano Lett. 2015;15(11):7388-93. https://doi.org/10.1021/acs.nanolett.5b02802.

35. Konishi K, Higuchi T, Li J, Larsson J, Ishii S, Kuwata-Gonokami M. Polarization-controlled circular second-harmonic generation from metal hole arrays with threefold rotational symmetry. Phys Rev Lett. 2014;112(13):135502.

36. Bomzon Z, Biener G, Kleiner V, Hasman E. Space-variant Pancharatnam-Berry phase optical elements with computergenerated subwavelength gratings. Opt Lett. 2002;27(13):1141-3.

37. Tymchenko M, Gomez-Diaz JS, Lee J, Nookala N, Belkin MA, Alu A. Gradient nonlinear pancharatnam-berry metasurfaces. Phys Rev Lett. 2015;115(20):207403.

38. Silveirinha M, Engheta N. Tunneling of electromagnetic energy through subwavelength channels and bends using epsilon-near-zero materials. Phys Rev Lett. 2006;97(15):157403.

39. Alu A, Silveirinha MG, Salandrino A, Engheta N. Epsilon-near-zero metamaterials and electromagnetic sources: Tailoring the radiation phase pattern. Phys Rev B. 2007;75(15):155410.

40. Edwards B, Alu A, Young ME, Silveirinha M, Engheta N. Experimental verification of epsilon-near-zero metamaterial coupling and energy squeezing using a microwave waveguide. Phys Rev Lett. 2008;100(3):033903.

41. Molesky S, Dewalt CJ, Jacob Z. High temperature epsilon-near-zero and epsilon-near-pole metamaterial emitters for thermophotovoltaics. Opt Express. 2013;21(1):A96-A110.

42. Niu XX, Hu XY, Chu SS, Gong QH. Epsilon-near-zero photonics: a new platform for integrated devices. Adv Opt Mater. 2018;6(10):1701292

43. Vassant S, Hugonin J-P, Marquier F, Greffet J-J. Berreman mode and epsilon near zero mode. Opt Express. 2012;20(21): 23971-7. https://doi.org/10.1364/OE.20.023971.

44. Campione S, Brener I, Marquier F. Theory of epsilon-near-zero modes in ultrathin films. Phys Rev B. 2015;91(12):121408.

45. Jia W, Liu M, Lu Y, Feng X, Wang $Q$, Zhang $X$, et al. Broadband terahertz wave generation from an epsilon-near-zero material. Light: Sci Appl. 2021;10(1):11.

46. Liberal I, Engheta N. Near-zero refractive index photonics. Nat Photonics. 2017;11(3):149.

47. Engheta N. Pursuing near-zero response. Science. 2013;340(6130):286

48. Reshef O, De Leon I, Alam MZ, Boyd RW. Nonlinear optical effects in epsilon-near-zero media. Nat Rev Mater. 2019;4(8): 535-51.

49. Kinsey N, DeVault C, Boltasseva A, Shalaev VM. Near-zero-index materials for photonics. Nat Rev Mater. 2019;4(12):742-60.

50. Tian W, Liang F, Chi S, Li C, Yu H, Zhang H, et al. Highly efficient super-continuum generation on an epsilon-near-zero surface. ACS Omega. 2020;5(5):2458-64. 
51. Niu X, Hu X, Sun Q, Lu C, Yang Y, Yang H, et al. Polarization-selected nonlinearity transition in gold dolmens coupled to an epsilon-near-zero material. Nanophotonics. 2020;9(16):4839-51.

52. Moitra P, Yang Y, Anderson Z, Kravchenko II, Briggs DP, Valentine J. Realization of an all-dielectric zero-index optical metamaterial. Nat Photonics. 2013:7(10):791-5.

53. Capretti A, Wang Y, Engheta N, Dal NL. Comparative study of second-harmonic generation from epsilon-near-zero indium tin oxide and titanium nitride nanolayers excited in the near-infrared spectral range. ACS Photonics. 2015;2(11): 1584-91.

54. Capretti A, Wang Y, Engheta N, Dal NL. Enhanced third-harmonic generation in Si-compatible epsilon-near-zero indium tin oxide nanolayers. Opt Lett. 2015;40(7):1500-3.

55. Luk TS, de Ceglia D, Liu S, Keeler GA, Prasankumar RP, Vincenti MA, et al. Enhanced third harmonic generation from the epsilon-near-zero modes of ultrathin films. Appl Phys Lett. 2015;106(15):151103.

56. Alam MZ, De Leon I, Boyd RW. Large optical nonlinearity of indium tin oxide in its epsilon-near-zero region. Science. 2016;352(6287):795-7. https://doi.org/10.1126/science.aae0330.

57. Caspani L, Kaipurath RP, Clerici M, Ferrera M, Roger T, Kim J, et al. Enhanced nonlinear refractive index in epsilon-nearzero materials. Phys Rev Lett. 2016;116(23):233901. https://doi.org/10.1103/PhysRevLett.116.233901.

58. Niu X, Hu X, Lu C, Sheng Y, Yang H, Gong Q. Broadband dispersive free, large, and ultrafast nonlinear material platforms for photonics. Nanophotonics. 2020;9(15):4609-18. https://doi.org/10.1515/nanoph-2020-0420.

59. Kuttruff J, Garoli D, Allerbeck J, Krahne R, De Luca A, Brida D, et al. Ultrafast all-optical switching enabled by epsilonnear-zero-tailored absorption in metal-insulator nanocavities. Commun Phys. 2020;3(1):114.

60. Jiang X, Lu H, Li Q, Zhou H, Zhang S, Zhang H. Epsilon-near-zero medium for optical switches in a monolithic waveguide chip at 1.9 um. Nanophotonics. 2018;7(11):1835-43. https://doi.org/10.1515/nanoph-2018-0102.

61. Chen P-Y, Argyropoulos C, Alu A. Enhanced nonlinearities using plasmonic nanoantennas. Nanophotonics. 2012;1(3-4): 221-33.

62. Kauranen M, Zayats AV. Nonlinear plasmonics. Nat Photonics. 2012;6(11):737-48. https://doi.org/10.1038/nphoton.2 012.244 .

63. Hou-Tong C, Antoinette JT, Nanfang Y. A review of metasurfaces: physics and applications. Rep Prog Phys. 2016;79(7): 076401.

64. Kuznetsov Al, Miroshnichenko AE, Brongersma ML, Kivshar YS, Luk'yanchuk B. Optically resonant dielectric nanostructures. Science. 2016;354(6314):2472.

65. Kruk S, Kivshar Y. Functional meta-optics and nanophotonics governed by Mie resonances. ACS Photonics. 2017:4(11): 2638-49. https://doi.org/10.1021/acsphotonics.7b01038.

66. Chang S, Guo X, Ni X. Optical metasurfaces: progress and applications. Annu Rev Mater Res. 2018;48(1):279-302.

67. Krasnok A, Tymchenko M, Alù A. Nonlinear metasurfaces: a paradigm shift in nonlinear optics. Mater Today. 2018;21(1):8-21.

68. Zou C, Sautter J, Setzpfandt F, Staude I. Resonant dielectric metasurfaces: active tuning and nonlinear effects. J Phys D Appl Phys. 2019;52(37):373002.

69. Pertsch T, Kivshar Y. Nonlinear optics with resonant metasurfaces. MRS Bull. 2020;45(3):210-20. https://doi.org/10.1557/ mrs.2020.65.

70. Terhune RW, Maker PD, Savage CM. Optical harmonic generation in calcite. Phys Rev Lett. 1962;8(10):404.

71. Kang L, Cui YH, Lan SF, Rodrigues SP, Brongersma ML, Cai WS. Electrifying photonic metamaterials for tunable nonlinear optics. Nat Commun. 2014;5:4680.

72. Lee K-T, Taghinejad M, Yan J, Kim AS, Raju L, Brown DK, et al. Electrically biased silicon metasurfaces with magnetic Mie sesonance for tunable harmonic generation of light. Acs Photonics. 2019;6(11):2663-70.

73. Chen S, Li KF, Li G, Cheah KW, Zhang S. Gigantic electric-field-induced second harmonic generation from an organic conjugated polymer enhanced by a band-edge effect. Light: Sci Appl. 2019;8(1):17.

74. Yap BK, Xia R, Campoy-Quiles M, Stavrinou PN, Bradley DDC. Simultaneous optimization of charge-carrier mobility and optical gain in semiconducting polymer films. Nat Mater. 2008;7(5):376-80. https://doi.org/10.1038/nmat2165.

75. Friberg S, Hong CK, Mandel L. Measurement of time delays in the parametric production of photon pairs. Phys Rev Lett. 1985:54(18):2011-3.

76. Hong CK, Ou ZY, Mandel L. Measurement of subpicosecond time intervals between 2 photons by interference. Phys Rev Lett. 1987;59(18):2044-6.

77. Bocquillon E, Couteau C, Razavi M, Laflamme R, Weihs G. Coherence measures for heralded single-photon sources. Phys Rev A. 2009;79(3):035801.

78. O'Brien JL, Furusawa A, Vučković J. Photonic quantum technologies. Nat Photonics. 2009;3(12):687-95.

79. Kwiat PG, Mattle K, Weinfurter H, Zeilinger A, Sergienko AV, Shih Y. New high-intensity source of polarization-entangled photon pairs. Phys Rev Lett. 1995;75(24):4337-41.

80. Kwiat PG, Waks E, White AG, Appelbaum I, Eberhard PH. Ultrabright source of polarization-entangled photons. Phys Rev A. 1999:60(2):R773-R6.

81. Kim T, Fiorentino M, Wong FNC. Phase-stable source of polarization-entangled photons using a polarization Sagnac interferometer. Phys Rev A. 2006;73(1):012316.

82. Yin J, Cao Y, Li Y-H, Liao S-K, Zhang L, Ren J-G, et al. Satellite-based entanglement distribution over 1200 kilometers. Science. 2017;356(6343):1180-4.

83. Okoth C, Cavanna A, Santiago-Cruz T, Chekhova MV. Microscale generation of entangled photons without momentum conservation. Phys Rev Lett. 2019;123(26):263602.

84. Marino G, Solntsev AS, Xu L, Gili VF, Carletti L, Poddubny AN, et al. Spontaneous photon-pair generation from a dielectric nanoantenna. Optica. 2019;6(11):1416-22.

85. Poddubny AN, lorsh IV, Sukhorukov AA. Generation of photon-plasmon quantum states in nonlinear hyperbolic metamaterials. Phys Rev Lett. 2016;117(12):123901.

86. Petrov MI, Nikolaeva AA, Frizyuk KS, Olekhno NA. Second harmonic generation and spontaneous parametric downconversion in Mie nanoresonators. J Phys: Conf Ser. 2018;1124:051021.

87. Song H, Nagatsuma T. Present and future of terahertz communications. IEEE Trans Terahertz Sci Technol. 2011; 1(1):256-63. 
88. Nagatsuma T, Ducournau G, Renaud CC. Advances in terahertz communications accelerated by photonics. Nat Photonics. 2016;10(6):371-9.

89. Wade CG, Šibalić N, de Melo NR, Kondo JM, Adams CS, Weatherill KJ. Real-time near-field terahertz imaging with atomic optical fluorescence. Nat Photonics. 2017;11(1):40-3.

90. Stantchev RI, Sun B, Hornett SM, Hobson PA, Gibson GM, Padgett MJ, et al. Noninvasive, near-field terahertz imaging of hidden objects using a single-pixel detector. Sci Adv. 2016;2(6):e1600190.

91. Mathanker SK, Weckler PR, Wang N. Terahertz (THz) applications in food and agriculture: a review. Trans ASABE. 2013; 56(3):1213-26.

92. Federici JF, Schulkin B, Huang F, Gary D, Barat R, Oliveira F, et al. THz imaging and sensing for security applications_-explosives, weapons and drugs. Semicond Sci Technol. 2005;20(7):S266-S80.

93. Liu J, Dai J, Chin SL, Zhang XC. Broadband terahertz wave remote sensing using coherent manipulation of fluorescence from asymmetrically ionized gases. Nat Photonics. 2010;4(9):627-31.

94. Xu W, Xie L, Zhu J, Xu X, Ye Z, Wang C, et al. Gold nanoparticle-based terahertz metamaterial sensors: mechanisms and applications. ACS Photonics. 2016;3(12):2308-14.

95. Nahata A, Weling AS, Heinz TF. A wideband coherent terahertz spectroscopy system using optical rectification and electro-optic sampling. Appl Phys Lett. 1996;69(16):2321-3.

96. Wu Q, Litz M, Zhang XC. Broadband detection capability of ZnTe electro-optic field detectors. Appl Phys Lett. 1996; 68(21):2924-6.

97. Hebling J, Almasi G, Kozma IZ, Kuhl J. Velocity matching by pulse front tilting for large-area THz-pulse generation. Opt Express. 2002;10(21):1161-6.

98. Yeh KL, Hoffmann MC, Hebling J, Nelson KA. Generation of $10 \mathrm{mu}$ J ultrashort terahertz pulses by optical rectification. Appl Phys Lett. 2007;90(17):171121.

99. Luo L, Chatzakis I, Wang J, Niesler FBP, Wegener M, Koschny T, et al. Broadband terahertz generation from metamaterials. Nat Commun. 2014;5(1):3055.

100. Keren-Zur S, Tal M, Fleischer S, Mittleman DM, Ellenbogen T. Generation of spatiotemporally tailored terahertz wavepackets by nonlinear metasurfaces. Nat Commun. 2019;10(1):1778.

101. Fang M, Shen NH, Sha WEl, Huang Z, Koschny T, Soukoulis CM. Nonlinearity in the dark: broadband terahertz generation with extremely high efficiency. Phys Rev Lett. 2019;122(2):027401.

102. Okawachi Y, Saha K, Levy JS, Wen YH, Lipson M, Gaeta AL. Octave-spanning frequency comb generation in a silicon nitride chip. Opt Lett. 2011;36(17):3398-400.

103. Jung $H$, Xiong $C$, Fong $K Y$, Zhang $X$, Tang HX. Optical frequency comb generation from aluminum nitride microring resonator. Opt Lett. 2013;38(15):2810-3.

104. Shen Y, Harris NC, Skirlo S, Prabhu M, Baehr-Jones T, Hochberg M, et al. Deep learning with coherent nanophotonic circuits. Nat Photonics. 2017;11(7):441-6.

105. Taghinejad M, Cai WS. All-optical control of light in micro- and nanophotonics. Acs Photonics. 2019;6(5):1082-93.

106. Almeida VR, Barrios CA, Panepucci RR, Lipson M. All-optical control of light on a silicon chip. Nature. 2004;431(7012): $1081-4$.

107. Pelc JS, Rivoire K, Vo S, Santori C, Fattal DA, Beausoleil RG. Picosecond all-optical switching in hydrogenated amorphous silicon microring resonators. Opt Express. 2014;22(4):3797-810.

108. Shcherbakov MR, Vabishchevich PP, Shorokhov AS, Chong KE, Choi DY, Staude I, Miroshnichenko AE, Neshev DN, Fedyanin AA, Kivshar YS. Ultrafast all-optical switching with magnetic resonances in nonlinear dielectric nanostructures. Nano Lett. 2015;15(10):6985-90. https://doi.org/10.1021/acs.nanolett.5b02989.

109. Wurtz GA, Pollard R, Hendren W, Wiederrecht GP, Gosztola DJ, Podolskiy VA, et al. Designed ultrafast optical nonlinearity in a plasmonic nanorod metamaterial enhanced by nonlocality. Nat Nanotechnol. 2011;6(2):106-10.

110. Guo PJ, Schaller RD, Ketterson JB, Chang RPH. Ultrafast switching of tunable infrared plasmons in indium tin oxide nanorod arrays with large absolute amplitude. Nat Photonics. 2016;10(4):267-73. https://doi.org/10.1038/nphoton.2016.14.

111. Clerici M, Kinsey N, DeVault C, Kim J, Carnemolla EG, Caspani L, et al. Controlling hybrid nonlinearities in transparent conducting oxides via two-colour excitation. Nat Commun. 2017:8:15829.

112. Yang YM, Kelley K, Sachet E, Campione S, Luk TS, Maria JP, et al. Femtosecond optical polarization switching using a cadmium oxide-based perfect absorber. Nat Photonics. 2017;11(6):390-5.

113. Alam MZ, Schulz SA, Upham J, De Leon I, Boyd RW. Large optical nonlinearity of nanoantennas coupled to an epsilonnear-zero material. Nat Photonics. 2018;12(2):79-83.

114. Voisin C, Del Fatti N, Christofilos D, Vallée F. Ultrafast electron dynamics and optical nonlinearities in metal nanoparticles. J Phys Chem B. 2001;105(12):2264-80.

115. Krasavin AV, Ginzburg P, Zayats AV. Free-electron optical nonlinearities in plasmonic nanostructures: a review of the hydrodynamic description. Laser Photonics Rev. 2018;12(1):1700082.

116. Del Fatti N, Bouffanais R, Vallee F, Flytzanis C. Nonequilibrium electron interactions in metal films. Phys Rev Lett. 1998; 81(4):922-5.

117. Ren MX, Jia BH, Ou JY, Plum E, Zhang JF, MacDonald KF, et al. Nanostructured plasmonic medium for terahertz bandwidth all-optical switching. Adv Mater. 2011;23(46):5540-4. https://doi.org/10.1002/adma.201103162.

118. Kinsey N, DeVault C, Kim J, Ferrera M, Shalaev VM, Boltasseva A. Epsilon-near-zero Al-doped ZnO for ultrafast switching at telecom wavelengths. Optica. 2015;2(7):616-22. https://doi.org/10.1364/OPTICA.2.000616.

119. Guo QB, Cui YD, Yao YH, Ye YT, Yang Y, Liu XM, et al. A solution-processed ultrafast optical switch based on a nanostructured epsilon-near-zero medium. Adv Mater. 2017;29(27):7.

120. Wang J, Coillet A, Demichel O, Wang Z, Rego D, Bouhelier A, et al. Saturable plasmonic metasurfaces for laser mode locking. Light Sci Appl. 2020;9:50.

121. Kartner FX, Jung ID, Keller U. Soliton mode-locking with saturable absorbers. IEEE J Selected Topics Quantum Electron. 1996;2(3):540-56. https://doi.org/10.1109/2944.571754.

122. Keller U, Weingarten KJ, Kartner FX, Kopf D, Braun B, Jung ID, et al. Semiconductor saturable absorber mirrors (SESAM's) for femtosecond to nanosecond pulse generation in solid-state lasers. IEEE J Selected Topics Quantum Electron. 1996; 2(3):435-53. 
123. Spuhler GJ, Paschotta R, Fluck R, Braun B, Moser M, Zhang G, et al. Experimentally confirmed design guidelines for passively Q-switched microchip lasers using semiconductor saturable absorbers. J Opt Soc Am B-Opt Phys. 1999;16(3): 376-88. https://doi.org/10.1364/JOSAB.16.000376.

124. Keller U. Recent developments in compact ultrafast lasers. Nature. 2003;424(6950):831-8.

125. Keller U, Tropper AC. Passively modelocked surface-emitting semiconductor lasers. Phys Rep-Rev Sect Phys Lett. 2006; 429(2):67-120.

126. Bao Q, Zhang H, Wang Y, Ni Z, Yan Y, Shen ZX, et al. Atomic-layer graphene as a saturable absorber for ultrafast pulsed lasers. Adv Funct Mater. 2009;19(19):3077-83.

127. Sun Z, Hasan T, Torrisi F, Popa D, Privitera G, Wang F, et al. Graphene mode-locked ultrafast laser. ACS Nano. 2010;4(2): 803-10.

128. Sun Z, Hasan T, Ferrari AC. Ultrafast lasers mode-locked by nanotubes and graphene. Physica E-Low-Dimensional Syst Nanostructures. 2012;44(6):1082-91.

129. Martinez A, Sun Z. Nanotube and graphene saturable absorbers for fibre lasers. Nat Photonics. 2013;7(11):842-5.

130. Wang K, Wang J, Fan J, Lotya M, O'Neill A, Fox D, et al. Ultrafast saturable absorption of two-dimensional MoS $_{2}$ nanosheets. ACS Nano. 2013;7(10):9260-7. https://doi.org/10.1021/nn403886t.

131. Woodward RI, Kelleher EJR, Howe RCT, Hu G, Torrisi F, Hasan T, et al. Tunable Q-switched fiber laser based on saturable edge-state absorption in few-layer molybdenum disulfide (MoS 2 ). Opt Express. 2014;22(25):31113-22.

132. Luo Z, Wu D, Xu B, Xu H, Cai Z, Peng J, Weng J, Xu S, Zhu C, Wang F, Sun Z, Zhang H. Two-dimensional material-based saturable absorbers: towards compact visible-wavelength all-fiber pulsed lasers. Nanoscale. 2016;8(2):1066-72. https:// doi.org/10.1039/C5NR06981E.

133. Li D, Jussila H, Karvonen L, Ye G, Lipsanen H, Chen X, et al. Polarization and thickness dependent absorption properties of black phosphorus: new saturable absorber for ultrafast pulse generation. Sci Rep. 2015;5:15899.

134. Wang Y, Huang G, Mu H, Lin S, Chen J, Xiao S, et al. Ultrafast recovery time and broadband saturable absorption properties of black phosphorus suspension. Appl Phys Lett. 2015;107(9):091905.

135. Hu G, Albrow-Owen T, Jin X, Ali A, Hu Y, Howe RCT, et al. Black phosphorus ink formulation for inkjet printing of optoelectronics and photonics. Nat Commun. 2017:8:278.

136. Krause JL, Schafer KJ, Kulander KC. High-order harmonic generation from atoms and ions in the high intensity regime. Phys Rev Lett. 1992;68(24):3535-8.

137. Schafer KJ, Yang B, DiMauro LF, Kulander KC. Above threshold ionization beyond the high harmonic cutoff. Phys Rev Lett. 1993;70(11):1599-602. https://doi.org/10.1103/PhysRevLett.70.1599.

138. Corkum PB. Plasma perspective on strong field multiphoton ionization. Phys Rev Lett. 1993;71(13):1994-7.

139. Stolow A, Bragg AE, Neumark DM. Femtosecond time-resolved photoelectron spectroscopy. Chem Rev. 2004;104(4): 1719-57. https://doi.org/10.1021/cr020683w.

140. Cavalieri AL, Mueller N, Uphues T, Yakovlev VS, Baltuska A, Horvath B, et al. Attosecond spectroscopy in condensed matter. Nature. 2007:449(7165):1029-32.

141. Corkum PB, Krausz F. Attosecond science. Nat Phys. 2007;3(6):381-7.

142. Krausz F, Ivanov M. Attosecond Phys. Rev Mod Phys. 2009;81(1):163-234.

143. Ferray M, Lhuillier A, Li XF, Lompre LA, Mainfray G, Manus C. Multiple-harmonic conversion of 1064-nm radiation in raregases. J Phys B-Atom Mol Opt Phys. 1988;21(3):L31-L5.

144. Baltuska A, Udem T, Uiberacker M, Hentschel M, Goulielmakis E, Gohle C, et al. Attosecond control of electronic processes by intense light fields. Nature. 2003;421(6923):611-5.

145. McFarland BK, Farrell JP, Bucksbaum PH, Gühr M. High harmonic generation from multiple orbitals in N2. Science. 2008; 322(5905):1232.

146. Kim S, Jin J, Kim Y-J, Park I-Y, Kim Y, Kim S-W. High-harmonic generation by resonant plasmon field enhancement. Nature. 2008;453(7196):757-60.

147. Ghimire S, DiChiara AD, Sistrunk E, Agostini P, DiMauro LF, Reis DA. Observation of high-order harmonic generation in a bulk crystal. Nat Phys. 2011;7(2):138-41.

148. Luu TT, Garg M, Kruchinin SY, Moulet A, Hassan MT, Goulielmakis E. Extreme ultraviolet high-harmonic spectroscopy of solids. Nature. 2015;521(7553):498-502.

149. Schubert O, Hohenleutner M, Langer F, Urbanek B, Lange C, Huttner U, et al. Sub-cycle control of terahertz highharmonic generation by dynamical Bloch oscillations. Nat Photonics. 2014;8(2):119-23.

150. Han S, Kim H, Kim YW, Kim Y-J, Kim S, Park I-Y, et al. High-harmonic generation by field enhanced femtosecond pulses in metal-sapphire nanostructure. Nat Commun. 2016;7(1):13105.

151. Vampa G, Ghamsari BG, Siadat Mousavi S, Hammond TJ, Olivieri A, Lisicka-Skrek E, et al. Plasmon-enhanced highharmonic generation from silicon. Nat Phys. 2017;13(7):659-62.

152. Liu H, Guo C, Vampa G, Zhang JL, Sarmiento T, Xiao M, et al. Enhanced high-harmonic generation from an all-dielectric metasurface. Nat Phys. 2018;14(10):1006-10.

153. Yang Y, Lu J, Manjavacas A, Luk TS, Liu H, Kelley K, Maria JP, Runnerstrom EL, Sinclair MB, Ghimire S, Brener I. Highharmonic generation from an epsilon-near-zero material. Nat Phys. 2019;15(10):1022-6. https://doi.org/10.1038/s41567019-0584-7.

154. Sivis M, Taucer M, Vampa G, Johnston K, Staudte A, Naumov AY, Villeneuve DM, Ropers C, Corkum PB. Tailored semiconductors for high-harmonic optoelectronics. Science. 2017;357(6348):303-6. https://doi.org/10.1126/ science.aan2395.

155. Wang J, Bo F, Wan S, Li W, Gao F, Li J, et al. High-Q lithium niobate microdisk resonators on a chip for efficient electrooptic modulation. Opt Express. 2015;23(18):23072-8.

156. Rao A, Patil A, Rabiei P, Honardoost A, Desalvo R, Paolella A, et al. High-performance and linear thin-film lithium niobate Mach-Zehnder modulators on silicon up to $50 \mathrm{GHz}$. Opt Lett. 2016;41(24):5700-3.

157. Liang H, Luo $R, H e Y$, Jiang $H$, Lin Q. High-quality lithium niobate photonic crystal nanocavities. Optica. 2017; 4(10):1251-8.

158. Zhang M, Wang C, Cheng R, Shams-Ansari A, Loncar M. Monolithic ultra-high-Q lithium niobate microring resonator. Optica. 2017;4(12):1536-7. 
159. Wang C, Zhang M, Chen X, Bertrand M, Shams-Ansari A, Chandrasekhar S, Winzer P, Lončar M. Integrated lithium niobate electro-optic modulators operating at CMOS-compatible voltages. Nature. 2018;562(7725):101-4. https://doi. org/10.1038/s41586-018-0551-y.

160. Wang C, Zhang M, Stern B, Lipson M, Loncar M. Nanophotonic lithium niobate electro-optic modulators. Opt Express. 2018;26(2):1547-55.

161. Gao BF, Ren MX, Wu W, Hu H, Cai W, Xu JJ. Lithium niobate metasurfaces. Laser Photonics Rev. 2019;13(5):6.

162. Vabishchevich PP, Liu S, Sinclair MB, Keeler GA, Peake GM, Brener I. Enhanced second-harmonic generation using broken symmetry III-V semiconductor Fano metasurfaces. ACS Photonics. 2018;5(5):1685-90. https://doi.org/10.1021/a csphotonics.7b01478.

163. Koshelev K, Tang Y, Li K, Choi D-Y, Li G, Kivshar Y. Nonlinear metasurfaces governed by bound states in the continuum. ACS Photonics. 2019;6(7):1639-44.

164. Liu Z, Xu Y, Lin Y, Xiang J, Feng T, Cao Q, et al. High-Q quasibound states in the continuum for nonlinear metasurfaces. Phys Rev Lett. 2019;123(25):253901.

165. Lin Z, Liang X, Loncar M, Johnson SG, Rodriguez AW. Cavity-enhanced second-harmonic generation via nonlinearoverlap optimization. Optica. 2016;3(3):233-8.

166. Hughes TW, Minkov M, Williamson IAD, Fan S. Adjoint method and inverse design for nonlinear nanophotonic devices ACS Photonics. 2018:5(12):4781-7.

167. Sitawarin C, Jin W, Lin Z, Rodriguez AW. Inverse-designed photonic fibers and metasurfaces for nonlinear frequency conversion invited. Photonics Research. 2018;6(5):B82-B9.

168. Lei X, Rahmani M, Yixuan M, Smirnova DA, Kamali KZ, Fu D, et al. Enhanced light-matter interactions in dielectric nanostructures via machine-learning approach. Adv Photonics. 2020;2(2):026003.

169. Shi L, Iwan B, Nicolas R, Ripault Q, Andrade JRC, Han S, et al. Self-optimization of plasmonic nanoantennas in strong femtosecond fields. Optica. 2017;4(9):1038-43.

170. Zimmermann P, Hötger A, Fernandez N, Nolinder A, Müller K, Finley JJ, et al. Toward plasmonic tunnel gaps for nanoscale photoemission currents by on-chip laser ablation. Nano Lett. 2019;19(2):1172-8.

171. Li Z-Z, Wang L, Fan H, Yu Y-H, Sun H-B, Juodkazis S, et al. O-FIB: far-field-induced near-field breakdown for direct nanowriting in an atmospheric environment. Light-Sci Appl. 2020;9(1):41.

172. Liu X-Q, Yu L, Yang S-N, Chen Q-D, Wang L, Juodkazis S, et al. Optical nanofabrication of concave microlens arrays. Laser Photonics Rev. 2019;13(5):1800272.

173. Liu X-Q, Yang S-N, Yu L, Chen Q-D, Zhang Y-L, Sun H-B. Rapid engraving of artificial compound eyes from curved sapphire substrate. Adv Funct Mater. 2019;29(18):1900037.

174. Wei D, Wang C, Wang H, Hu X, Wei D, Fang X, et al. Experimental demonstration of a three-dimensional lithium niobate nonlinear photonic crystal. Nat Photonics. 2018;12(10):596-600.

\section{Publisher's Note}

Springer Nature remains neutral with regard to jurisdictional claims in published maps and institutional affiliations.

\section{Submit your manuscript to a SpringerOpen ${ }^{\circ}$ journal and benefit from:}

- Convenient online submission

- Rigorous peer review

- Open access: articles freely available online

- High visibility within the field

- Retaining the copyright to your article

Submit your next manuscript at $\boldsymbol{\nabla}$ springeropen.com 